\title{
Le droit pénal et la covid-19 en République Démocratique du Congo.
}

\author{
Pour une politique pénale de reconnaissance active des droits de \\ l'homme.
}

\author{
Par Jean Pierre KABEMBA KAPENGA*
}

\section{Résumé}

La lutte contre la pandémie de Covid-19 est un défi de survie que chaque État du monde s'est fixé. Cette lutte passe inévitablement par la mise en place de règles strictes à respecter, dont la violation s'accompagne de sanctions. Or, en RDC, entre logique pénale et logique administrative, le choix est fait sur la répression administrative, dont la mise en œuvre n'a pas répondu au besoin de dissuasion souhaité dans cette lutte. Or, cette voie est l'expression de la mise à l'écart du droit pénal, pourtant indispensable pour assurer la contrainte et la dissuasion.

Ainsi, après avoir décrit les problèmes posés dans la gestion de la pandémie, cette étude propose une nouvelle stratégie basée sur la prise en compte d'une politique pénale de reconnaissance active des droits de l'homme comme voie de sortie. Ceci n'exclut en rien l'aspect pénal (qui devra être réorganisé) des postulats de prise en compte des besoins fondamentaux de la population dans la lutte contre le Covid-19.

\begin{abstract}
The fight against the Covid-19 pandemic is a survival challenge that every State in the world has set for itself. This fight inevitably involves the implementation of strict rules to be observed, the violation of which is accompanied by sanctions. However, in the DRC, between penal logic and administrative logic, the choice is made on administrative repression, the implementation of which has failed to meet the need for the deterrence desired in this fight. However, this way is an expression of the sidelining of the criminal law, yet essential to ensure the constraint and the dissuasion.

Thus, after having described the problems posed in the management of the pandemic, this study proposed a new strategy based on taking into account a criminal policy of active recognition of human rights as a way out.. This does not at all exclude the penal aspect

* L'auteur est avocat au Barreau de Kinshasa/Matete en RDC, apprenant en troisième cycle à l'Université de Kinshasa et chercheur en droit. Il est auteur d'un article publié dans la revue de recherche et de documentation juridique d'Afrique, en 2020 intitulé Rationalité pénale à l'épreuve des attentes de la population en RDC : un procès fait au système pénal congolais. Il est aussi candidat assistant à l’université de Kinshasa. Adresse e-mail : aryankabemba@gmail.com.
\end{abstract}


(which will have to be reorganized) postulates for taking into account the basic needs of the population in the fight against Covid-19.

\section{INTRODUCTION}

Il y a bien longtemps, Jean-Jacques Rousseau écrivait que tout Etat libre où les grandes crises n'ont pas été prévues, est à chaque orage en danger de péril ${ }^{1}$. Cette pensée a pris corps dans le contexte congolais où, comme partout ailleurs, des circonstances exceptionnelles ont prévalu à la mise en œuvre des mesures, mieux, d'un régime presqu'exceptionnel.

En effet, la pandémie à covid- $19^{2}$ a secoué la quiétude du monde entier, mettant la population de la majorité des pays dans une situation précaire de limitation substantielle de plusieurs droits fondamentaux au profit de la survie. La République démocratique du Congo n'a pas, du reste, échappé à cette situation pour le moins exceptionnelle dans la mesure où l'Etat d'urgence sanitaire a été décrété par le Chef de l'Etat par l'ordonnance n `20/014 du 24 mars 2020 portant proclamation de l'état d'urgence sanitaire pour faire face à l'épidémie de Covid-19, et renouvelé six fois de suite pour être levée, dans une adresse faite par le chef de l'Etat, le 21 juillet 2020.

Cependant, durant la période de la Covid-19 en RDC (première vague ${ }^{3}$ ), la répression ne s'est pas sentie directement au départ. Cette situation est due à l'indisponibilité de la législation pouvant permettre de mettre en cause les auteurs des comportements qui violent les consignes ou les mesures de confinement. Des messages de sensibilisation ont pris cours, invitant la population au respect des mesures-barrières. Qui plus est, l'ordonnance $n$ `20/014 du 24 mars 2020 portant proclamation de l'Etat d'urgence sanitaire pour faire face à l'épidémie de Covid-19 prévoit, en son article 3, une série des mesures relatives à l'exercice de la liberté notamment l'interdiction de tous les voyages de la capitale vers les provinces et vice-versa, les rassemblements, réunions et célébrations de plus de 20 personnes, la fermeture provisoire des écoles, des universités, des instituts supérieurs, etc. Plus récemment, dans le cadre de la deuxième vague de la Covid-19, l'arrêté ministériel $\mathrm{n}$ ○25/CAB/VPM/MINTERSECAC/GKM/032/2020 du 17 décembre 2020 portant modalités pratiques d'exécution des mesures de limitation contre la deuxième vague de la pandémie Covid-19 a été pris dans la même logique que les précédents textes. Hormis un panel d'in-

1 JJ. Rousseau, « considération sur le gouvernement de Pologne et sur sa réformation projetée », in collections complètes œuvres, Genève 1780-1789, vol.1, p. 480.

2 C'est une maladie respiratoire provoquée par un coronavirus émergent, le SARS-CoV-2. L'épidémie a débuté dans la ville de Wuhan, en Chine, fin décembre 2019 et s'est rapidement propagée dans le monde entier.

3 A suivre cette terminologie dans les médias et les discours publics, la première vague de la Covid-19 est partie de la découverte du patient 0 de la RDC le 10 mars 2020, elle s'est poursuivie jusqu'à la levée de l'Etat d'urgence. La deuxième vague a commencé avec la recrudescence des cas de Covid-19 à Kinshasa et s'est matérialisée à partir de l'ordonnance du chef de l'Etat annonçant des nouvelles mesures à observer dans la lutte contre la Covid-19 le 16 décembre 2020. 
terdictions qui sont prises en termes de mesures de prévention de la pandémie, de manière générale, aucune sanction pénale ou administrative n'est prévue contre les contrevenants aux mesures édictées. Toutefois, il faut reconnaitre que l'arrêté $n$ ${ }^{\circ} \mathrm{SC} / 022 / \mathrm{CAB} / \mathrm{GVK} / \mathrm{GNM} / 2020$ du 18 avril 2020du gouverneur de province, prévoit le paiement d'une amende qui sanctionne le non-respect de la mesure relative au port obligatoire des masques ${ }^{4}$.

La logique de la réaction sociale à tirer de ce dernier texte est une répression administrative. Les amendes y prévues sont prononcées et appliquées, avec beaucoup d'abus déplorés $^{5}$, par la police administrative ${ }^{6}$. Si cette manière peut être envisagée de par sa souplesse par rapport à la procédure pénale, sa mise en œuvre n'a pas permis la dissuasion ${ }^{7}$ qui est pourtant l'effet attendu des mesures de contrainte édictées durant cette période ${ }^{8}$. En effet, l'apparition de la pandémie a généré, au début, une peur de l'inconnu qui a permis sur le coup le respect des conseils donnés par les pouvoirs publics. On pouvait observer des dispositifs d'hygiène à toutes les entrées des magasins, des immeubles de bureau; des contrôles aux entrées avec la prise de température. Peu après, il s'est fait sentir un relâchement des mesures barrières édictées, jusqu'à la levée de l'état d'urgence sanitaire par le Président. Pourtant, comme il l'a souligné dans son discours à la nation diffusé à la RTNC le 22 juillet 2020, « il doit être clairement entendu que la fin de l'état d'urgence ne veut pas dire la fin de l'épidémie ».

Malheureusement, depuis lors, les gestes barrières ne sont plus respectés par une importante frange de la population. Dans le secteur de l'enseignement, le non-respect des mesures barrières est perceptible à plusieurs égards. La Radio Okapi rapporte que "plusieurs étudiants qui fréquentent les instituts supérieurs et les universités ne portent pas de masques. Ils sont collés les uns aux autres dans leurs auditoires $»{ }^{9}$.

Des cinq gestes barrières connus dans la lutte contre le coronavirus, seule la prise de température à l'entrée de certains établissements rappelle de l'existence de la pandémie. A l'université de Kinshasa, toutes les entrées sont libres d'accès. De notre constat sur les lieux, il ressort que la seule différence entre la période de la covid-19 et celle normale est la

4 Article 2 de l'arrêté nºSC/022/CAB/GVK/GNM/2020 du 18 avril 2020.

5 Voir infra.

6 Toute amende n'est pas pénale. Est une amende administrative, celle qui est prononcée par une autorité administrative. Le critère organique en ce que l'amende pénale (peine d'amende) doit être prononcée par le juge est celui qui est retenu par la doctrine. Lire à ce sujet la définition donnée par le Professeur SITA MUILA A., Manuel de droit pénal général congolais, éd. L'Harmattan, Paris, 2020, p. 274. Dans le même sens, lire Emmanuel Ronsenfeld et Jean Veil, Sanctions administratives, sanctions pénales, Dans le Seuil, n¹28, 2009, p. 63.

7 Par la peur qu'elle implique, la peine dissuade les citoyens de « délinquer » ou de « redélinquer ». Lire Gérard Lopez \& Stamatios Tzitzis (Dir), Dictionnaire des sceinces criminelles, Paris, 2004, p. 688.

8 Voir infra.

9 www.radiokapi.net/2020/9/23, consulté le 7 janvier 2021. 
séparation des entrées pendant la première entre celle du personnel scientifique (professeurs, chefs des travaux et assistants) et celle des étudiants.

Dans les marchés publics, le constat est le même. Les vendeurs comme les acheteurs ainsi que certains agents de l'ordre se collent les uns aux autres sans aucun respect de la distanciation. Le nombre des fréquentations est généralement élevé, et le port des masques est carrément aléatoire de part et d'autres. Le slogan est que la pandémie n'existe pas. C'est ainsi que témoignent des personnes interviewées dans le même sens déclarant pour un d'entre eux : " je porte le masque pour éviter les tracasseries de la police, la maladie n'existe pas dans notre pays », et pour une vendeuse des vivres frais au marché du rondpoint Ngaba : " ici chez nous la vie continue comme d'habitude, nous travaillons tous les jours sans nous soucier de la maladie. Notre peur provient du comportement des agents de la police qui peuvent parfois nous brutaliser en cas de non-port de masque ». Il en transpire un manque de dissuasion.

Il y a aussi le cas des transports en commun où le risque de contamination est trop élevé. Une dizaine des personnes collées entre elles, dont la plupart ne portent pas de masques et ce, sous le regard impuissant et complice des agents de l'ordre, peut-on observer dans les rues de Kinshasa.

Des cas sont légion, on pourrait les démontrer dans tous les secteurs de la vie en RDC. Nous partons dès lors de l'hypothèse selon laquelle il existe une série d'obstacles juridiques et factuels qui empêchent la réalisation de la dissuasion comme objectif pénal. Or, nous pensons que la dissuasion devrait être le but poursuivi par la contrainte dans un contexte de l'urgence pour permettre, en l'occurrence, de limiter la propagation de la pandémie.

C'est ainsi que cette étude, par une démarche à la fois théorique (recherche documentaire) et empirique (recherche qualitative) au-delà de démontrer les obstacles dont question, propose une série des solutions à tous les problèmes ainsi posés (obstacles) en amont.

\section{A. Des obstacles à la dissuasion dans la gestion de la Covid-19 en RDC}

Un panel d'obstacles à la réalisation de la dissuasion peut être mis en évidence, notamment le théorique et pratique de gestion de la Covid-19 en RDC qui n'est pas adapté à cette situation exceptionnelle (I), la méconnaissance de la place du droit pénal dans la gestion de la pandémie (II), la répression administrative comme modalité de gestion de la pandémie qui n'a pas manqué d'accuser des limites si bien que des abus ont vite fait surface (III) et enfin, le contexte de précarité et de sous-développement dans lequel se trouve depuis des lustres la société congolaise constitue un obstacle majeur à la mise en œuvre de cet objectif pénal dans la gestion de la Covid-19 (IV).

\section{L'inadéquation du système pénal en vigueur à la situation de la pandémie}

La Covid-19 est une situation inédite en RDC. Elle est apparue soudainement et a surpris la société entière qui a procédé par des tâtonnements dans tous les domaines dans la façon de 
gérer la crise sanitaire ainsi générée. Elle a trouvé une société sans défense, qui ne s'était pas préparée à affronter un adversaire de si grande taille, surtout inconnue du grand public.

Le droit congolais n'a pas échappé à cette réalité qui n'a fait qu'apporter des problèmes majeurs au droit auxquels il faut trouver des solutions adéquates. Trois sortes de problèmes se posent essentiellement en ce qui concerne le droit pénal :

Premièrement, nous relevons l'indisponibilité des normes de base pouvant permettre une mise en œuvre de l'objectif pénal durant la période de l'urgence. En effet, en vue de respecter les mesures barrières, un certain nombre de comportements devraient tomber sous le coup de la loi pénale. C'est le cas d'une personne qui présente des symptômes mais qui refuse de se présenter devant un médecin en mettant délibérément autrui en danger; l'inaction de l'autorité publique qui laisse des groupes de gens se réunir sans imposer le respect des mesures barrières; la violation des règles de l'isolement par un malade; la célébration et les réunions de plus de 20 personnes; etc. Pourtant, en l'état actuel des choses, la plupart des mesures prises ne sont pas pénales. Les sanctions y relatives sont administratives. Or, nul juriste ne doute vraiment que pour être efficace, une règle bien pensée doit être assortie d'une sanction adaptée. Et le droit pénal, étant souvent présenté comme le bras armé des autres matières, il n'y a rien de surprenant à requérir que le dispositif spécial à mettre en ouvre dans le cadre du régime de crise soit assorti des sanctions pénales ${ }^{10}$. Il faut reconnaitre que le respect des mesures barrières édictées par l'autorité publique devait nécessairement être soumis à des contraintes pénales.

Deuxièmement, l'insuffisance du dispositif existant dans ce sens que, certaines situations pourtant vitales n'ont pas eu de modalité de gestion prédéfinies. En effet, seul l'arrêté du gouverneur précité prévoit le paiement d'une amende de 5000 francs en cas de non port de masque. Pourtant, ce n'est pas la seule interdiction qui est prévue durant cette période de la pandémie. D'autres ne sont généralement pas assorties des sanctions si bien que les agents de police sont obligés d'improviser. C'est ainsi qu'un vendeur de boisson dans un bar (barman) qui se trouve à Mont-Ngafula, au niveau de la commune déclare : "les policiers ne savent pas trop quoi faire. C'est pour cela qu'ils nous ravissent nos chaises quand on dépasse le nombre de personnes prévu ou quand on va au-delà du couvre-feu ». On peut retenir là que les mesures édictées, les interdictions faites ne sont pas accompagnées des sanctions à même de répondre au besoin de dissuasion.

Toutefois, il faut reconnaitre que certains comportements liés à la pandémie peuvent être poursuivis sur base des incriminations existantes. Nous pouvons noter le cas de la tromperie sur la qualité de la chose vendue (la vente des faux gels hydroalcooliques par exemple) ${ }^{11}$, le vol ${ }^{12}$ des masques, le vol des matériels médicaux destinés à la prise en

10 David Riccardi, « l'adaptation du droit répressif à l'épidémie de covid-19: histoire et enjeux d'une quête redoutable ", droit et coronavirus. Le droit face aux circonstances exceptionnelles, RDLF $2020, n^{\circ} 35$.

11 Articles 99 et 100 du code pénal congolais.

12 Article 79 du code pénal congolais. 
charge des patients atteints du virus, etc. cependant, leur prise en charge ne peut pas constituer un dispositif suffisant pour limiter la pandémie de la Covid-19.

Troisièmement, il convient de nous interroger sur la possibilité de l'application pratique des règles pénales durant la période de la Covid-19 au regard du formalisme procédural en vigueur. En effet, «dans notre pays, entre le moment où un délinquant est arrêté et celui où intervient la sanction, il s'écoule un laps de temps tellement long que la peine intervient dans l'ignorance ou l'indifférence totale de l'opinion publique. Il est évident que, considérée sous cet angle, la peine perd sa raison d'être sociale aux yeux de l'homme congolais $»^{13}$. Et pourtant le but poursuivi par la matière pénale dans ce domaine est la dissuasion des auteurs des comportements qui facilitent la propagation de la pandémie, et, à ce titre, l'action pénale doit être assez forte pour décourager mais aussi assez rapide pour limiter les casses et permettre l'amélioration rapide de la situation sanitaire ${ }^{14}$. La précarité institutionnelle fait de la justice congolaise une justice artisanale dans laquelle les nouvelles technologies de l'information et de la communication sont quasi-inexistantes. Et pourtant, la Covid-19 est opposée à l'idée de se réunir parce qu'il y a risque de contamination.

Il est donc évident que le recours rigoureux au système pénal existant ne peut pas faciliter la dissuasion qui est poursuivie dans le cadre de la lutte contre la Covid-19.

\section{Méconnaissance de la place du droit pénal dans la gestion de la Covid-19}

La covid-19 a mis en danger la santé d'un grand nombre au point que toute la société est ébranlée par sa soudaine apparition. C'est ici le lieu d'user des fonctions axiologique et répressive du droit pénal. En effet, celui-ci protège les valeurs essentielles de la société et consolide sur la base de cette protection l'ensemble du système juridique ${ }^{15}$. A travers cette fonction, le législateur identifie et sélectionne les valeurs sociales qu'il juge indispensable de défendre par la coercition pénale ${ }^{16}$. En outre, le droit pénal réprime les infractions commises et permet la réparation des dommages causés à la victime. Cette vue répressive met l'accent sur la fonction de création des incriminations ou des infractions par la détermination des comportements interdits ou prohibés, et sur celle de distribution des châtiments aux malfaiteurs et aux hors-la-loi ${ }^{17}$.

Pourtant, dans son action, l'autorité congolaise a adopté une logique administrative, sans doute moins dissuasive et surtout avec beaucoup d'abus qui ont été observés ${ }^{18}$. Dans

13 Luzolo BAMBI LESSA E.J., Manuel de procédure pénale, Kinshasa, 2011, p. 8.

14 David Riccardi, op.cit.

15 SITA MUILA A., Le droit pénal et la famille, essai d'analyse systémique et axiologique, tome 1, Thèse pour le doctorat en droit, université de droit, d'économie et des sciences politiques d'AixMarseille, 2001, page d'épigraphe.

16 SITA MUILA A., Manuel de droit pénal général congolais, op.cit., p. 83.

17 Pierre AKELE ADAU et SITA MUILA A., Les crimes contre l'humanité en droit congolais, Kinshasa, 1999, p. 6.

18 Voir infra. 
son arrêté du 18 avril 2020, l'autorité urbaine rendait déjà obligatoire le port de masques, assortissant le non-respect de cette mesure par une amende de 5000 francs congolais.

Ce texte qui a permis aux policiers d'exiger le payement de l'amende démontre à suffisance le choix fait entre la logique pénale et la logique administrative. Il s'agit là des amendes administratives tant il est vrai qu'elles ne sont pas prononcées par le juge pénal. Il faudrait préciser cependant que cette manière de gérer les violations des textes pendant la période de la Covid-19 est certes expéditive et souple dans la mesure où la répression est automatique et ne demande pas la lourdeur d'une procédure pénale, mais elle a été jonchée de plusieurs autres problèmes non-résolus, débouchant parfois même à des violations des droits de l'homme. Il s'est vite observé une banalisation sans égal des mesures qui ont été prescrites dans le cadre de la lutte contre la Covid-19. Seule la conscience de chacun pouvait le pousser à adopter telle ou telle autre attitude sans soucier de l'impact qu'un comportement fautif pourrait avoir sur la santé des autres.

\section{Des solutions discriminatoires et liberticides dans la gestion de la Covi-19}

Le choix opéré entre le droit pénal et la répression administrative a brillé par ses effets contradictoires avec l'objectif poursuivi, à savoir la dissuasion. Les modalités juridiques de gestion de la Covid-19 ont accusé une certaine marginalisation des catégories défavorisées dans un registre purement prescriptif (1) et, théoriquement, un recul de certaines garanties fondamentales des personnes (2).

\section{De la protection à la marginalisation}

Françoise Digneffe enseigne, parlant de considérer tout individu comme acteur dans une situation problématique déterminée, « qu'il faut retrouver dans l'histoire du sujet, ce qui peut représenter un enjeu pour lui compte tenu des ressources dont il dispose et de ses capacités à se représenter le jeu dans lequel il joue $»{ }^{19}$. Ceci signifie, le cas échéant, que l'action gouvernementale doit tenir compte des capacités individuelles de chacun à s'adapter aux mesures prises. Dans la gestion de la pandémie à Covid-19, une volonté a été exprimée par les pouvoirs publics de préserver la population de la contamination. A cette occasion, des mesures ont été prises comme dit-haut, mais reste à s'interroger sur l'efficacité de ce dispositif. Dans leur application, des violations des droits de l'homme ontété constatées à plusieurs égards. A Kinshasa, l'amende de 5000Fc assortie au non-respect de l'obligation du port de masque est généralement accompagnée d'une brutalité policière que certains protagonistes dénoncent. Un Kinois déclare : «je ne voulais pas au fait porter ça, mais si je le porte, c'est parce que lorsqu'on ne le porte pas la police agresse, surtout en ce moment où on est

19 Françoise Digneffe, « le concept d'acteur social et le sens de son utilisation dans les théories criminologiques ", in Acteur social et délinquance. Une grille de lecture du système de justice pénale, en hommage au professeur Christian Debuyst, Liège, Bruxelles, 1990, p. 353. 
en train de vivre la misère $»^{20}$. Un autre s'interrogeait : "ils veulent qu'on regarde (respecte) leur loi, mais est-ce que cette loi nous regarde? ». L'organisation nommée la Fondation congolaise pour la promotion des droits humains et la paix déclare que «c'est une décision illégale et non conforme aux lois de la République Démocratique du Congo. C'est une décision qui ne vise qu'à cautionner l'anarchie et les violations des droits de l'homme. Tous les comportements des policiers, des arrestations, des tortures qu'ils infligent aux paisibles citoyens sont des actes arbitraires et qui doivent être sanctionnés sévèrement conformément aux lois en vigueur $»^{21}$.

Bien-sûr que la loi ne respecte pas la population. Il y a là un système de gestion d'une situation qui est fait en dehors de ses destinataires, leur imposant juste des contraintes en cas de l'inobservance. Evidemment que des cas d'inobservance ont été observés mais sans doute parce qu'il est impossible de parvenir à une solution par un mécanisme qui est mis en place sans tenir compte de la situation de précarité sociale, des inégalités économiques et qui ne considère pas l'individu comme un acteur dans la gestion de la pandémie.

Nous pouvons ainsi en déduire que l'action policière et même gouvernementale, dans un cadre tout à fait prescriptif, ne cherche pas à donner sens à l'acte, elle ne situe pas l'individu dans un contexte déterminé. Ce qui conduit tout naturellement à la mise à l'écart des $a$ priori, ici, des droits collectifs de la personne qui ne sont pas pris en compte au profit des contraintes prescrites par une catégorie déterminée.

\section{Recul des garanties pénales fondamentales des personnes}

Des arrestations ont été effectuées en vue de répondre aux besoins essentiels de la lutte contre la Covid-19. Selon les agents de la police, les arrestations effectuées contre les personnes qui violaient les règles étables en confinement étaient nécessaires parce que justifiées par le souci préserver la santé de tous. Une nécessité que nous partageons également, mais reste à se demander la légitimité du dispositif.

En effet, le principe de légalité des délits et des peines prévu à l'article 17 de la Constitution du 18 février 2006 consacre une sécurité juridique contre l'arbitraire. Malheureusement celui-ci a été largement bafoué. Ce principe postule que seul le législateur dispose du monopole d'incriminer un comportement donné et cela d'une manière claire et précise. Il doit établir des textes précis quant à la définition des infractions et à la détermination des sanctions $^{22}$. Le dispositif qui a donné le pouvoir aux policiers de réprimer le non-respect des mesures barrières n'a pas tenu compte de cette exigence. On pourrait comprendre que la situation de la Covid-19 est inédite et a été soudaine, mais il ne faut pas perdre de vue que « si grave que soit la situation d'une société au regard de la criminalité, toutes les mesures visant à la combattre qui ne tiendraient pas compte des valeurs démocratiques, des droits de

20 Disponible sur www.google.com/amp/s/amp.dw, consulté le 13 décembre 2020.

21 Idem.

22 NYABIRUNGU MWENE SONGA, op.cit., p. 38. 
l'homme et de la prééminence du droit seraient inacceptables $»^{23}$. Encore, même en matière administrative, le principe de légalité exige que les sanctions à prendre par l'autorité administrative doivent être préalablement définies. Malheureusement, comme dit haut, les interdictions faites ne sont toutes pas assorties des sanctions si bien que les policiers improvisent. Le témoignage de ce vendeur de boissons qui déclare que les policiers ravissent des chaises à titre de sanction; ou de ce motard qui parle de passer nuit à la belle étoile avec les policiers sont des cas flagrants de l'atteinte à ce principe.

En outre, le principe de nécessité de la loi pénale a été méconnu. En effet, ce principe prévoit l'obligation du législateur d'incriminer les actes qui portent atteinte aux valeurs essentielles de la société. Il est tenu d'intervenir chaque fois que des intérêts aussi essentiels que la santé et la liberté sont mis en cause. Son silence dans cette matière où des comportements bien précis, notamment le fait d'adopter volontairement un comportement imprudent qui génère un risque de contamination (comme le fait pour une personne qui se sait positive de ne pas respecter l'isolement), pourraient porter atteinte aux valeurs de la société est un manquement flagrant à son obligation et donc une violation du principe de nécessité de la loi.

\section{Le permanent sous-développement économique et social}

La RDC est l'un des pays les plus pauvres du monde. Avec un budget annuel pour l'année 2020 d'environ 6,8 milliards de dollars américains (réduit de $43 \%$ ) pour une population qui avoisine les 80.000 .000 d'habitants (quatre-vingt millions d'habitants), la galère ne peut que se percevoir déjà dans les chiffres. La ville de Kinshasa, épicentre de la pandémie, renferme à peu près 14 millions d'habitants pour 9000 kilomètres carrés.

Le magazine le Point rapporte que : "Employeur de plus de $77 \%$ des congolais et pourvoyeur de revenus à plus de $90 \%$ des ménages de la RDC, le secteur informel de l'économie congolaise constitue l'épine dorsale de survie. Or, le fonctionnement de ce secteur est fondamentalement social et dicté par la mobilité humaine (urbaine et rurale). Les mesures barrières recommandées pour lutter contre la pandémie condamnent presqu'au suicide ce secteur, et avec lui les ménages qui en dépendent $»^{24}$.

En effet, une grande partie de cette population vit des « shida » ou « coop ». Ce sont des travaux journaliers qui requièrent nécessairement que la ville soit vivante, qu'il y ait de la circulation et que les personnes dépensent. On peut y trouver des vendeurs ambulants des produits de première nécessité, des cireurs des souliers, des chauffeurs de taxi, des vendeurs dans des marchés, des commissionnaires, etc. Les revenus de ces catégories ne leur permettent pas de réaliser des économies pouvant leur permettre de tenir longtemps enfermés. En outre, lorsqu'on restreint leur temps de travail, il leur est difficile de gagner suffisam-

23 Conseil d'Europe, Recommandation n R(96)8(1996).

24 Par Junior Malula, publié le 20 août 2020 et disponible sur www.lepoint.fr, consulté le 7 janvier 2021. 
ment de quoi tenir une journée. Sans compter sur le coût des matériels de protection (gel et masque de protection) sur ce petit peuple qui vit au jour le jour. Dans pareille circonstance, les mesures relatives à la quarantaine, à la distanciation sociale, le confinement, le couvrefeu, etc., ne peuvent pas aisément être respectées.

En outre, l'Unicef rapporte que le manque d'eau en RDC est un facteur du non-respect des mesures barrières dans ce sens que le nouveau coronavirus est une épidémie qui demande beaucoup en ce qui concerne les mesures d'hygiène. Parmi ces mesures, la règle maitresse nous exige de nous laver les mains ${ }^{25}$. Et pourtant, l'eau est une ressource qui pose beaucoup de problèmes dans les grandes villes de la RDC, particulièrement à Kinshasa où la pénurie d'eau est un autre mal à côté de la pandémie ${ }^{26}$.

La plupart des droits collectifs sont bafoués en RDC. L'eau, l'électricité, l'emploi, l'environnement sain, etc. sont des luxes à Kinshasa, surtout dans certaines contrées. Dans un tel contexte, il est difficile d'interdire à quelqu'un de se promener, ou de lui restreindre le temps de travail sans tomber sur les récalcitrants. En suspendant certaines activités des personnes sans leur trouver des équivalents ou des palliatifs, on ne peut pas résoudre efficacement un problème.

D'où, cette situation de pauvreté devient un obstacle majeur mettant les agents dans un état permanent de nécessité.

\section{B. Options fondamentales de la reforme}

Le temps et l'urgence de la situation constituent le vecteur essentiel dans la recherche du régime répressif à établir durant la période de la pandémie. Le but recherché par ce régime sera essentiellement la dissuasion rapide afin de permettre l'amélioration des conditions de lutte contre la pandémie. Et pourtant, les longues procédures dans une situation qui n'a pas vocation à durer en soi ne sont pas favorables à la dissuasion. Dès lors, il faudrait un montage juridique à même de préserver les droits fondamentaux du mieux que possible, mais surtout de garantir une gestion efficace de la pandémie à Covid-19.

Cependant, la gestion en cours a démontré des limites, certes surmontables, de notre système pénal en vigueur. L'indisponibilité de certaines règles de droit pénal pourtant essentielles, et les difficultés pratiques de mise en œuvre du dispositif existant sont des éléments qui sous-tendent l'inadéquation du système pénal congolais à la pandémie de la Covid-19. En conséquence, l'on a constaté un recul de la place du droit pénal dans la gestion de la pandémie, occasionnant des multiples violations des droits de l'homme et la méconnaissance de fait de certains principes de droit pénal.

Il nous revient ainsi de trouver une juste mesure entre la rigueur et l'importance des principes fondamentaux du droit pénal et la nécessité de lutter contre la pandémie en sacri-

25 Unicef, disponible sur reliefweb.int/report/..., page consultée le 7 janvier 2021.

26 CICR RDC disponible sur www.google.com, page consultée le 7 janvier 2021. 
fiant ou en assouplissant certains des principes ci-haut cités. Notre démarche consiste à privilégier le volet préventif, avec un faible recours au droit pénal.

Dans cette démarche, il faudra mobiliser les efforts plusieurs intervenants, en commençant par le législateur, passant par les institutions administratives pour finir par la population.

\section{La part du législateur}

Le législateur congolais doit mieux s'outiller pour déterminer et gérer la criminalité qui résulte de la pandémie ou qui se commet à l'occasion de celle-ci. L'action du législateur se situe ainsi à deux niveaux :

\section{La criminalisation de certains comportements}

Hormis des comportements infractionnels commis qui ont un support légal en droit congolais, il existe d'autres qui ne sont pas pris en compte par le législateur. Nous pouvons citer par exemple la violation des règles de confinement. A ce sujet, l'individu qui va quitter la quarantaine ou l'isolement en dépit de l'interdiction y relative pourra se voir sanctionner des peines qui seront déterminées par le texte; le refus de délibéré de déclarer un cas suspect ou confirmé par un médecin ou par toute personne disposant de la faculté de dénicher la maladie sachant que ce refus met en danger plusieurs personnes; etc.

Aussi, faudra-t-il que le législateur puisse instituer une responsabilité nouvelle, la responsabilité de l'autorité publique. En effet, l'attitude de banalisation des mesures barrières peut occasionner la responsabilité pénale de l'autorité publique dès lors qu'il est établi, d'une part, qu'il y a eu propagation de la pandémie par sa négligence (ici par exemple le fait pour l'autorité compétente de ne pas prendre des dispositions pour isoler les malades ou de les laisser librement circuler), et, d'autre part, qu'il avait un pouvoir de le faire (empêcher la propagation). Il faut noter cependant que c'est un cas de discussion qui peut aboutir à des reformes mieux pensées. Le champ reste ouvert pour mûrir la réflexion sur cette forme de responsabilité en vue de pousser les autorités publiques à prendre au sérieux leurs fonctions dans la préservation des intérêts publics.

Plusieurs autres comportements entrent en ligne de compte pour une action législative. Il revient au législateur de les identifier et de les criminaliser pour une meilleure gestion de la Covid-19.

\section{L'institution d'une théorie de circonstances exceptionnelles en droit pénal}

Compte tenu du caractère exceptionnel et urgent de la situation de laquelle nous plonge la pandémie à Covid-19, la théorie de circonstances exceptionnelles devra déroger au droit commun à plusieurs égards. Des concessions doivent être faites en vue d'adapter le droit pénal congolais aux nécessités de lutte contre la pandémie. 
En premier lieu, nous citons le principe de légalité qui doit subir un assouplissement considérable. En effet, selon Beccaria, « le droit de faire des lois ne peut résider que dans la personne du législateur, qui représente toute la société unie par un contrat social ${ }^{27}$. Cette exigence est légitime au regard de la séparation des pouvoirs qui attribue le pouvoir des faire des lois au législatif ${ }^{28}$. Sans prétendre débattre sur la teneur des termes légalité et textualité ou encore normativité, nous pensons que le législateur congolais devrait déléguer son pouvoir de détermination des incriminations, et dans le cas des circonstances exceptionnelles, même des sanctions à l'autorité administrative (ou exécutive), de manière modérée et limitée.

Des limitations porteront nécessairement sur la gravité des infractions à incriminer et la portée de la sanction à prévoir pour un comportement déterminé. L'autorité exécutive devra se limiter à incriminer des comportements mineurs.

En deuxième lieu, nous avons le principe du procès équitable ou le droit à un procès équitable qui devra subir certains arrangements. Dans l'hypothèse d'un droit pénal exceptionnel, la loi pourra permettre à l'administration d'appliquer des peines dès lors qu'une constatation matérielle de l'infraction est établie. Il s'agira donc des infractions purement matérielles qui consistent dans la simple inobservation d'une prescription légale ou réglementaire. Elles ne supposent ni intention de violer la loi pénale, ni même imprudence ou négligence. Elles sont constituées dès que le fait réprimé par la loi est matériellement constaté ${ }^{29}$.

Cependant, la nature de ces infractions aux règles de confinement (qui sont purement matérielles) permet donc à l'administration d'appliquer la sanction (souvent l'amende pénale, et dans quelques rares cas, la servitude pénale) sans recourir à la longue procédure devant un juge, l'objectif étant ici d'assouplir la procédure afin d'obtenir un résultat efficace (la dissuasion).

En troisième lieu, la redéfinition de la récidive légale en ce qui concerne les infractions aux règles de confinement. En effet, la récidive peut être comprise comme la situation dans

27 Cesare BECCARIA, Des délits et des peines, Paris 2002, p. 14.

28 Cependant, cette rigueur qui s'attache à la forme du texte incriminateur, tel que précisé ci-haut (loi votée au parlement), parait de plus en plus dépassée aujourd'hui. En effet, l'article 153 alinéa 4 de la Constitution de la RDC du 18 février 2006 donne pouvoir aux cours et tribunaux d'appliquer les traités et accords internationaux dûment ratifiés. C'est ainsi qu'à l'heure actuelle, les traités qui ne sont pas des lois votées au parlement prévoient dans bien de cas des infractions que le juge congolais est tenu d'appliquer en vertu de la disposition constitutionnelle susmentionnée. Néanmoins, en droit français la constitution prévoit même le règlement comme une source de la norme pénale. Face à cette évolution constitutionnelle, certains auteurs comme Claude LOMBOIS proposent de retenir non plus le principe de la légalité, mais plutôt celui de la textualité. A ce propos, le professeur NYABIRUNGU, emboitant leurs pas, pense avec raison qu' " il est donc concevable et admissible, compte tenu de la complexité et de technicité de notre temps, que le législateur délègue son pouvoir, par nécessité et de manière limitée, de détermination des incriminations, à l'exclusion des sanctions. Celles-ci n'étant pas tributaires des contingences extérieures, leur détermination ne peut appartenir qu'au législateur ».

29 A.SITA MUILA, Manuel de droit pénal général congolais, op.cit., p. 213. 
laquelle se trouve une personne qui, déjà condamnée par une décision définitive passée en force de chose jugée pour avoir commis une infraction de gravité déterminée, en commet une nouvelle de même gravité endéans un délai déterminé par le législateur ${ }^{30}$. Les conditions de la récidive tiennent donc à la nature des infractions commises et au délai écoulé entre les deux infractions. Et la loi établit limitativement ces conditions.

Cependant, dans le cas des circonstances exceptionnelles, la gradation de la sanction sur base de la répétition ou la réitération d'un comportement prohibé devra suivre un autre cours que celui dicté par la loi ordinaire. Et donc, le délai et la nature des infractions devront être revus pour permettre aux pouvoirs publics de sanctionner une personne qui tombe sous le coup de la nouvelle récidive. Dans cette hypothèse, il pourra y avoir récidive même si le premier fait constaté n'a pas été condamné par un jugement définitif.

\section{L'action administrative, point focal de la réaction sociale à la pandémie}

Pour qu'il y ait application de la sanction, il faudrait que la violation d'une règle de droit soit illégitime. Cependant, lorsque le contexte socio-économique ne permet pas à la population de vivre pendant longtemps confinée, les interdictions posées ne pourront pas être respectées. Le meilleur moyen de faire respecter les normes ou les règles, c'est de prévenir le manquement à celles-ci d'une part en créant les conditions qui éliment les causes d'autre part, en créant un contrôle social qui garantisse leur application et enfin en éduquant et persuadant ou en rééduquant ${ }^{31}$.

A ce propos, le cas d'un confinement prolongé pendant une durée relativement longue, avec interdiction de sortie dans une ville où les mécanismes de livraison à domicile n'existent presque pas, et dans une société où une grande partie de la population vit au jour le jour par des travaux journaliers est plus qu'éloquent sur la mauvaise politique entreprise. Le confinement risque de régler un problème par un autre, empêchant la propagation de la pandémie mais laissant la population mourir de faim et de soif dans leurs maisons.

C'est ainsi que les politiques de gestion de la pandémie doivent nécessairement tenir compte des politiques sociales en ce qui concerne l'approvisionnement des personnes en eau, en électricité, en denrées alimentaires et en tous autres besoins de première nécessité. Le choix politique opéré sur un besoin doit inéluctablement être nécessaire et sinéquanone dans le but qu'il devra atteindre. C'est ici la part de l'administration qui devra définir des stratégies efficaces et efficientes pour rendre compte des besoins les plus légitimes de la population dans cette période rude.

Quoiqu'il en soit, la mesure de l'action administrative devra être le respect des droits de l'homme, conduisant dès lors les détenteurs du pouvoir public à adopter une politique active de reconnaissance des droits fondamentaux, où l'Etat tente de répondre aux besoins de

30 Idem, p. 288.

31 A. MALANGU MUABILA et J.P. KABEMBA KAPENGA, "Rationalité pénale à l'épreuve des attentes de la population en République démocratique du Congo : un procès fait au système pénal congolais », in RDJA, n95-2020, p. 405. 
sécurité de tous et non seulement aux besoins de sécurité contre le comportement délictueux de certaines personnes ${ }^{32}$. Il s'agit de la prévention même de la délinquance qui passe par la mise en place des politiques sociales des reconnaissances des droits collectifs et individuels.

\section{La population, un acteur important dans la lutte contre la pandémie}

Combattre la Covid-19 n'est pas qu'une affaire du législateur et des autres institutions. Une part non négligeable revient à la population qui est le destinataire même de toutes les mesures prises à tous égards.

En effet, si les Etats Unis d'Amérique ont enregistré le plus lourd bilan dans le monde ce n'est sûrement pas parce qu'ils manquaient de politiques sociales et pénales, moins encore de matériel médical. La faille est plutôt humaine, voire organisationnelle.

En effet, la discipline et le respect des mesures barrières doivent être inculqués à tout prix à la population qui devra s'abstenir d'adopter des comportements interdits sous peine d'exposer un plus grand nombre de personnes à la maladie. Tout le monde doit concourir à limiter la propagation de la pandémie en adoptant un comportement responsable dans la situation actuelle. C'est ici qu'une campagne de sensibilisation s'impose comme un devoir de tout citoyen prêtant main forte à la lutte entreprise pour éradiquer cette pandémie.

\section{Conclusion}

La situation de la pandémie à Covid-19 est une première dans l'histoire juridique de la RDC. Elle est apparue de manière soudaine et a donné lieu à des divers tâtonnements conséquemment à l'indisponibilité des modalités de gestion adaptées à la situation en cause. C'est dans la fièvre de ces engouements que nous avons éprouvé plusieurs inquiétudes notamment l'inadéquation du système pénal (ici le système pénal est pris comme une modalité à part de régulation sociale) aux besoins des circonstances (dissuasion), la méconnaissance de la place du droit pénal par le recours à la répression administrative, et la marginalisation ou la discrimination opérée par les pouvoirs publics sur une catégorie des personnes généralement dans une situation de faiblesse par rapport à leurs droits collectifs qui sont bafoués.

Cette étude constitue un repère pour une adaptation du droit pénal congolais à des circonstances exceptionnelles mettant le défi entre la protection des droits fondamentaux et l'amélioration des conditions de vie dans une situation de péril. Certes, l'apport du droit pénal dans la gestion de la pandémie n'a pas été pris à juste valeur, et les mesures édictées n'ont pas été respectées. On a assisté à une sorte de guéguerre entre les agents de l'ordre et la population qui a estimé que les policiers en faisaient trop, et que les mesures édictées ne tenaient pas compte de la situation intrinsèque des destinataires. 
Il donc clair qu'hormis la question de l'adaptation du droit pénal aux circonstances exceptionnelles, il s'est posé celui de l'opportunité du droit pénal dans un contexte de précarité constante où il est difficile de mettre en œuvre les règles pénales contre les personnes qui sont en état de nécessité permanent. Il revient donc aux pouvoirs publics de mettre en œuvre une politique pénale qui associe les droits de l'homme dans la recherche de la solution pénale. C'est une politique active de reconnaissance des droits collectifs qui doit précéder toute répression. Sans cela, la logique pénale ne sera que vaine et facteur de plusieurs autres problèmes à résoudre à l'avenir.

C'est dire la non satisfaction de ces droits est une cause de conflits dans les interactions sociales, alors même que la question des situations à risque (logement, emploi, nourriture, etc) auxquelles sont confrontées les personnes vulnérables n'entre pas en compte dans les politiques pénales. C'est ici la place des droits de l'homme dans les réponses pénales à donner dans la lutte contre la pandémie à Covid-19 dans la mesure où il faut une organisation sociale qui prend en compte les droits humains, la solidarité de tous et non la marginalisation de celui qui a posé un acte problématique. Dans cette conception, le système pénal qui n'est pas exclu ne doit plus être le principe, mais plutôt l'exception, réduit au strict nécessaire (en cas des graves violations). Le revers de cette reconnaissance c'est l'établissement des devoirs de chacun au profit de la collectivité. C'est là la philosophie africaine qui donne de la liberté à un individu responsable ${ }^{33}$, c'est-à-dire qui a non seulement des droits, mais aussi des devoirs à accomplir pour la collectivité. C'est une conception qui tire son fondement du sens communautaire de l'Afrique ${ }^{34}$.

\section{Bibliographie}

\section{A. Ouvrages}

1. AKELE ADAU Pierre et SITA MUILA A., Les crimes contre l'humanité en droit congolais, éd. Du CEPAS, Kinshasa, 1999.

2. BALANDA MIKWIN LELIEL, "African charter ou Human and people's Rights », dans K. CONTHER \& BENEDEK, New perspectives and conceptionsof international law. An afro-european dialogue, New-York, 1983.

3. BECCARIA Cesare, Des délits et des peines, éd. Du Boucher, Paris, 2002.

4. Conseil d'Europe, Recommandation $n^{\circ} \mathrm{R}(96) 8(1996)$.

5. LOPEZ Gérard \& STAMATIOS TZITZIS (Dir), Dictionnaire des sciences criminelles, Paris, Dalloz, 2004.

33 BALANDA MIKWIN LELIEL, "African charter ou Human and peoples Rights ", dans K. CONTHER \& BENEDEK, New perspectives and conceptionsof international law. An afro-eropean dialogue, New-York, 1983, p. 137.

34 Raoul KIENGE KIENGE INTUDI, « l'application de la Convention internationale relative aux droits de l'enfant en Afrique, Kinshasa, 2007, p. 22. 
6. KIENGE KIENGE INTUDI Raoul, L'application de la Convention internationale relative aux droits de l'enfant en Afrique, Kinshasa, 2007.

7. NYABIRUNGU Mwene SONGA, Traité de droit pénal général, 2è éd., DES, 2007.

8. SITA MUILA A., Manuel de droit pénal général congolais, éd. L'Harmattan, Paris, 2020.

9. TULKENS Françoise, Criminologie et droits humains, une rencontre indispensable, SD.

B. Articles

1. DIGNEFFE Françoise, « le concept d'acteur social et le sens de son utilisation dans les théories criminologiques ", in Acteur social et délinquance. Une grille de lecture du système de justice pénale, en hommage au professeur Christian Debuyst, Liège, Bruxelles, 1990.

2. MALANGU MUABILA A. et KABEMBA KAPENGA J.P., « Rationalité pénale à l'épreuve des attentes de la population en République démocratique du Congo : un procès fait au système pénal congolais », in RDJA, n95-2020.

3. RICCARDI David, «L'adaptation du droit répressif à l'épidémie de covid-19: histoire et enjeux d'une quête redoutable ", droit et coronavirus. Le droit face aux circonstances exceptionnelles, in RDLF 2020, n³5.

4. ROSENFELD Emmanuel et VEIL Jean, Sanctions administratives, sanctions pénales, Le Seuil, n¹28, 2009.

5. ROUSSEAU Jean-Jacques, « Considération sur le gouvernement de Pologne et sur sa réformation projetée », in collections complètes æuvres, Genève 1780-1789, vol.1.

C. Thèse

1. SITA MUILA A., Le droit pénal et la famille, essai d'analyse systémique et axiologique, tome 1, Thèse pour le doctorat en droit, université de droit, d'économie et des sciences politiques d'Aix-Marseille, 2001. 